\title{
On MEMS Reliability and Failure Mechanisms
}

\author{
Daniel J. Fonseca and Miguel Sequera \\ Department of Mechanical Engineering, The University of Alabama, Tuscaloosa, Al 35487, USA \\ Correspondence should be addressed to Daniel J. Fonseca, dfonseca@eng.ua.edu
}

Received 15 June 2011; Revised 9 August 2011; Accepted 1 September 2011

Academic Editor: Suk joo Bae

Copyright ( 2011 D. J. Fonseca and M. Sequera. This is an open access article distributed under the Creative Commons Attribution License, which permits unrestricted use, distribution, and reproduction in any medium, provided the original work is properly cited.

\begin{abstract}
Microelectromechanical systems (MEMS) are a fast-growing field in microelectronics. MEMS are commonly used as actuators and sensors with a wide variety of applications in health care, automotives, and the military. The MEMS production cycle can be classified as three basic steps: (1) design process, (2) manufacturing process, and (3) operating cycle. Several studies have been conducted for steps (1) and (2); however, information regarding operational failure modes in MEMS is lacking. This paper discusses reliability in the context of MEMS functionality. It also presents a brief review of the most relevant failure mechanisms for MEMS.
\end{abstract}

\section{Introduction}

Microelectromechanical systems (MEMS) are a relatively new and fast-growing field in microelectronics. MEMS are commonly used as actuators, sensors, and radio frequency and microfluidic components, as well as biocomposites, with a wide variety of applications in health care, automotive, and military industries. It is expected that the market for MEMS will grow to over $\$ 30 \mathrm{~B}$ by 2050 [1].

The MEMS lifecycle can be divided into three basic steps: (1) the design process, (2) the manufacturing process, and (3) the operating cycle. Several research studies have been conducted for the design and manufacturing of MEMS; however, information regarding failure analysis for MEMS can still be considered in its infancy stage [2].

There is a need to develop new tools and methodologies to understand the behavior of MEMS devices for distinct applications and operation conditions. MEMS are extremely diverse and their failure modes can be unique under different conditions [3].

MEMS represent a technology that can be defined as miniaturized mechanical and electromechanical elements (i.e., devices and structures) that are made using the techniques of microfabrication. Dimensions of MEMS devices can vary from well below one micron on the lower end of the dimensional spectrum, all the way to several millimeters [4].
Likewise, the types of MEMS devices can vary from relatively simple structures having no moving elements, to extremely complex electromechanical systems with multiple moving elements under the control of integrated microelectronics. A main criterion of MEMS is that there are at least some elements having some sort of mechanical functionality, whether or not these elements can move [4]. MEMS are manufactured using batch fabrication techniques similar to those used for integrated circuits. Unprecedented levels of functionality, reliability, and sophistication can be placed on a small silicon chip at a relatively low cost [1].

The real potential of MEMS starts to become fulfilled when these miniaturized sensors, actuators, and structures can all be merged onto a common silicon substrate along with integrated circuits (i.e., microelectronics). While the electronics are fabricated using integrated circuit (IC) process sequences (e.g., CMOS, bipolar, or BICMOS processes), the micromechanical components are fabricated using compatible "micromachining" processes that selectively etch away parts of the silicon wafer, or add new structural layers, to form the mechanical and electromechanical devices. MEMS can be merged not only with microelectronics, but with other technologies such as photonics. This is sometimes called "heterogeneous integration." Clearly, these technologies are filled with numerous commercial opportunities [4]. 


\section{Previous Studies on MEMS Reliability}

2.1. MEMS Reliability. Reliability for MEMS devices is identified as the next manufacturers challenge for the forthcoming years due to a growing market and stricter government safety regulations. It is necessary to understand several variables to have an approach of their behavior and functionality.

Very high levels of reliability are required in most industrial applications, such as automotive. For example, the automotive industry is now focused on failure rates lower than 10 FIT. 1 FIT is the usual unit corresponding to a proven failure over 109 hours [2]. In this context, several steps must be developed to understand internal variables (i.e., technologies related) and external variables (i.e., environment and operation conditions).

In automotive applications, MEMS reliability analysis is extremely important to identify and understand the different failure mechanisms that can be implicit such as mechanical, thermal, and chemical aspects related to the diversity of materials that can be used. Recent studies expect airbag penetration to increase from 40 to 60 million vehicles over the next five years (i.e., $80 \%$ of cars worldwide) [5].

MEMS devices require interaction with the environment to perform their mission. For instance, applications in automotive as well as high-demand manufacturing systems represent environments and surroundings result aggressive for MEMS, and thus, they require special attention. Standardized testing of MEMS components is partially covered in the Society of Automotive Engineers and the military via SAE J1221, SAE J575G, and Military Standard 750. These standards detail accelerating testing such as high- and lowtemperature storage, temperature cycling, and thermal shock [5].

In general, the automotive and manufacturing industries require most MEMS lifetime from five to ten years (or 100,000 to 150,000 miles on desert, tropical, or arctic locations in the case of automotive applications). For commercial trucks components, ten years or 1 million miles of problem free use are required [5].

2.2. Previous Efforts on MEMS Reliability. One of the most critical points in developing a reliability analysis is to understand the way in which a system can fail, or commonly known as its "root cause." For that reason, a failure mode is defined as the apparent failure of a system, and the failure mechanism as the physical cause (mechanical, chemical, or thermal) of the failure modes in the system [6]. Bhushan [4] emphasized that we have to start with a clear distinction between failure modes and failure mechanisms in MEMS.

Tanner et al. [7] evaluated the effect of humidity, vibrations, and shock environments in microelectro mechanical systems. Also, failure mechanisms and failure modes for each condition were discussed and analytical data used in the experiments. Moreover, Vallett [8] introduced state-ofthe-art microelectronic failure analysis processes, instrumentation, and principles. The major limitations and future prospects determined from industry roadmaps were discussed by the author.
Walraven [3] emphasized the significant success in MEMS products from a reliability perspective and categorized the following taxonomy groups to address their reliability concerns.

(i) Class I: no moving parts (pressure sensors and microphones).

(ii) Class II: moving parts, no rubbing or impacting surfaces (gyroscopes and RF oscillators).

(iii) Class III: moving parts with impacting surfaces.

(iv) Class IV: moving parts with impacting and rubber surfaces.

In addition, Walraven [9] briefly stated that some failure mechanisms described earlier would affect an MEMS device regardless of its class. Failures due to stiction and particle contamination have been shown to cause failure in all 4 classes of devices. Other investigators used Walraven [9] taxonomy groups classification such as Tanner [10] who also concluded that these classes typically share failure mechanisms and increase complexity.

Materials can be considered critical for their behavior under extreme conditions such as high temperatures, humidity, and vibration. Sharpe and Bagdahn [11] identified three general categories for mechanical properties analysis of MEMS: (1) elasticity from an applied force or vice versa, (2) inelastic device behavior, and (3) materials strength to set operating limits. Results of measurements of metals mechanical properties used in MEMS as well as references on materials and tests of interest were discussed. Additionally, specific data on important material such as DLC (diamondlike-carbon), nickel and nickel-iron, and polysilicon properties were analyzed in depth.

Several studies have been conducted to understand MEMS failures depending on the material composition, especially in silicon, which is commonly used for MEMS. Shea [12] discussed MEMS failure mechanisms and failure modes for space applications. Also, he explained reliability concerns under special environmental conditions such as radiation, vacuum, and thermal-vibration shocks. Ritchie et al. [13] examined the premature fatigue failure of siliconbased micron-scale structures for MEMS, and the fracture properties of mineralized tissue, specifically human bone. Fitzgerald et al. [14] described and validated a general methodology to predict the reliability of single-crystal silicon MEMS devices. This methodology used experimental data generated from fracture testing specimens combined with finite element modeling to predict the fracture probability for any MEMS device under any loading.

Pomeroy et al. [15] developed a dynamic-stress analysis method, based on time resolved micro-Raman spectroscopy, for reliability studies of microelectromechanical systems. Also, Jadaan et al. [16] designed a probabilistic Weibull methodology to understand the behavior and mechanical properties of MEMS brittle materials. This investigation concluded that a Weibull probabilistic method is applicable at the MEMS scale size, and that provides significant prediction data of their short- and long-term behavior. 


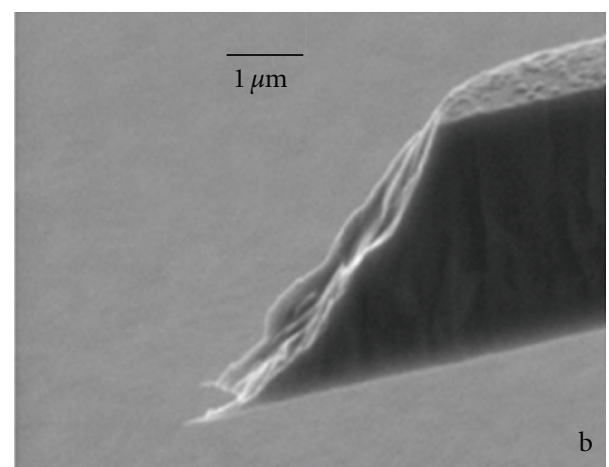

FIgURE 1: Surface fracture (courtesy of Sandia National Laboratories).

Starman et al. [17] investigated the measurement of residual and induced stress in an MEMS micromirror flexure utilizing micro-Raman spectroscopy. The investigation showed that micro-Raman spectroscopy can be used as an effective measurement technique to determine local and induced stress values in MEMS devices. Schwalke et al. [18] investigated the breakdown of extra thick gate oxides (50$150 \mathrm{~nm}$ ) used in power MOS device. Weibull probability plots were used to describe the failure distribution of the thick gate oxides. Luo et al. [19] examined some fundamental reliability aspects of high-k film through ramp voltage stress testing. By studying dielectric relaxation, and analyzing the transient conductivity, breakdown modes of the tested high$\mathrm{k}$ film were identified; a sensitive method of breakdown detection in ramped voltage tests was then proposed.

Accelerated testing has also been used to determine materials properties and measure reliability. Brown et al. [20] developed a resonant fatigue accelerated testing to demonstrate a failure mode that was previously unknown. Their work indicated that moisture can decrease the lifetime of cyclically stressed polysilicon components.

\section{MEMS Failure Mechanisms}

A critical part of understanding the reliability of any system comes from understanding the possible ways in which the system may fail. In MEMS, there are several failure mechanisms that have been found to be the primary sources of failure within devices $[21,22]$.

3.1. Mechanical Fracture. Mechanical fracture is defined as the breaking of a uniform material into two separate sections. In MEMS, it usually leads to the catastrophic failure of the device, although there are some structures that may have moderate performance degradations. No matter what the actual outcome, any fracturing is a serious reliability concern (Figure 1) [21, 23].

There are three types of fractures: ductile, brittle, and intercrystalline fractures. Ductile fracture, as the name implies, occurs in ductile materials. It is characterized by almost uninterrupted plastic deformation of a material. It is

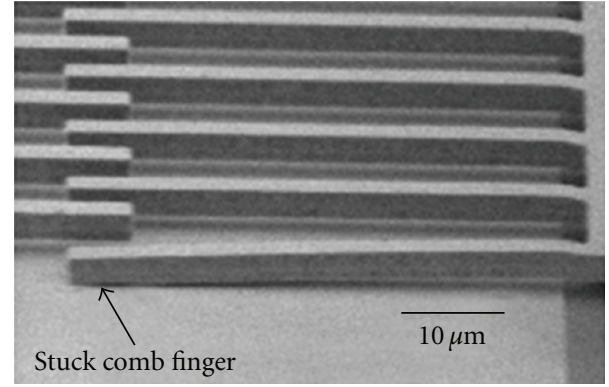

FIgure 2: Stiction in MEMS fingers (courtesy of Sandia National Laboratories).

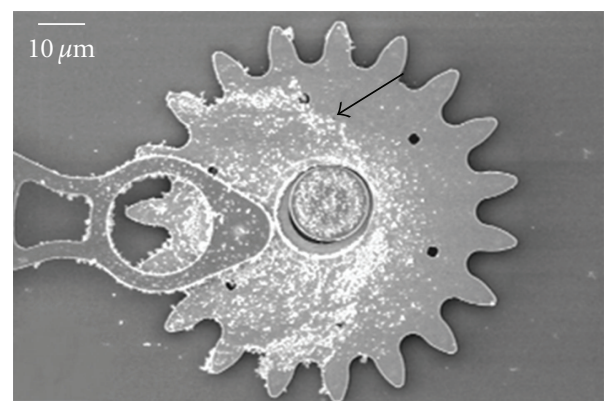

FIGURE 3: Wear debris in microengine (courtesy of Sandia National Laboratories).

usually signified by the necking, or extreme thinning, of a material at one specific point. Brittle fracture occurs along crystal planes and develops rapidly with little deformation. Intercrystalline fracture is a brittle fracture that occurs along grain boundaries in polycrystalline materials, often beginning at a point where impurities or precipitates accumulate. For MEMS, the latter two types of fracture are more common [21]. The stress levels that most accelerometer devices are subjected to are far lower than the breaking strength of the material used to build the mechanical structure $[6,24]$.

3.2. Stiction. Stiction is considered one of the most important problems in MEMS. Internal MEMS structures are so small that surface forces cause microscopic structures to stick together when their surfaces come into contact (see Figure 2). The most important surfaces forces are forces due to capillary condensation, van der Waals molecular forces, and chemical and hydrogen bonds between the surfaces. Surfaces tend to stick together when they are dried after the released etch [4].

3.3. Wear. Wear is caused by the motion of one surface over another. It is defined as the removal of material from a solid surface as the result of mechanical action [25]. Wear is generally considered an undesirable effect in MEMS. There are four main processes that cause wear, those are adhesion, abrasion, corrosion, and surface fatigue [21]. Figure 3 shows the wear debris on the surface of a microengine operated to 600,000 cycles (courtesy of Sandia National Laboratories). 


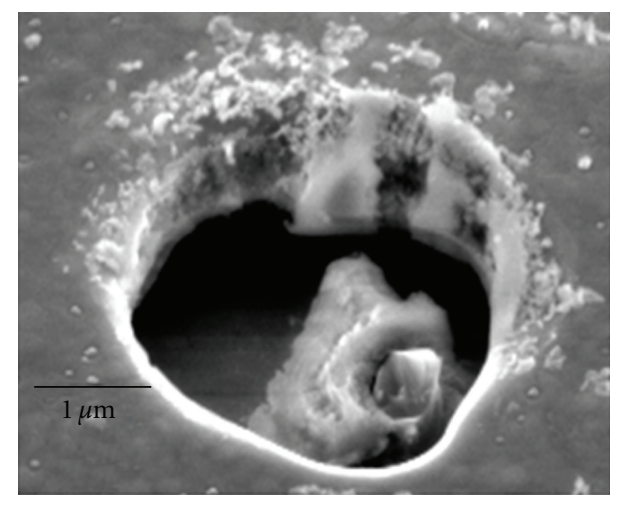

FIgURE 4: Surface wear in drive gears (courtesy of Sandia National Laboratories).

Adhesive wear is caused by one surface pulling fragments off of another surface while they are sliding. This is caused by surface forces bonding two materials together. When the bonds break, they are unlikely to separate at the original interface, which fractures one of the materials. Initial studies on the long-term effects of adhesive wear have been completed, with some interesting results being discovered [21].

Abrasive wear occurs when a hard, rough surface slides on top of a softer surface and strips away underlying material. While less prevalent in MEMS than adhesive wear, it can occur if particulates get caught in microgears and can tear apart a surface. Corrosive wear occurs when two surfaces chemically interact with one another and the sliding process strips away one of the reaction products. This type of wear could cause failure in chemically active MEMS. Certain types of microfluidic systems and biological MEMS are susceptible to corrosive wear. Corrosive wear is dependent upon the chemical reactions involved $[21,26]$.

Surface fatigue wear occurs mostly in rolling applications, such as bearings and gears. It affects highly polished surfaces that roll instead of sliding. Over time, the continued stressing and unstressing of the material under the roller will cause the appearance of fatigue cracks. These cracks then propagate parallel to the surface of a structure, causing material to flake off the surface. Surface fatigue wear tends to generate much larger particles than other wear mechanisms, with flakes as large as $100 \mathrm{~nm}$ being common in macroscopic applications $[25,27]$. Figure 4 shows a surface wear in a drive gear.

3.4. Delamination. A delamination condition occurs when a material interface loses its adhesive bond. It can be induced by a number of means, from mask misalignments to particulates on the wafer during processing. It can also arise as the result of fatigue induced by the long-term cycling of structures with mismatched coefficients of thermal expansion. No matter what the actual cause, the effects of delamination can be catastrophic. If the material is still present on the device, it can cause shorting or mechanical impedance $[21,28]$.

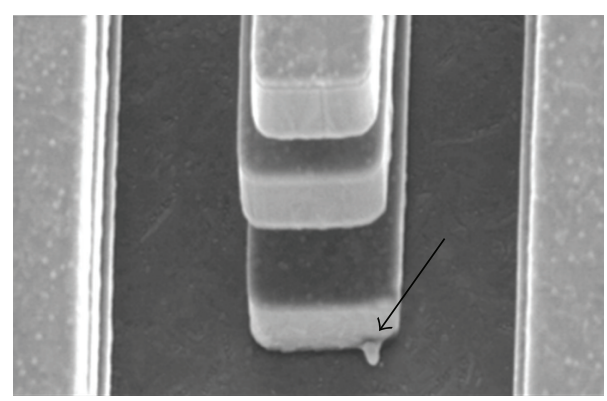

FIGURE 5: ESD failure in electrostatic actuator (courtesy of Sandia National Laboratories).

3.5. Vibration and Shocks. Vibration is a large reliability concern in MEMS. Due to the sensitivity and fragile nature of many MEMS, external vibrations can have disastrous implications. Either through inducing surface adhesion or through fracturing device support structures, external vibration can cause failure. Long-term vibration can also contribute to fatigue [9]. In contrast, shocks differ from vibration in that a shock is a single mechanical impact instead of a rhythmic event. A shock creates a direct transfer of mechanical energy across the device. Shocks can lead to both adhesion and fracture. Shocks can also cause wire bond shearing, a failure mode common to all semiconductor devices [4].

3.6. Electrostatic Discharge and Dielectric Charging. Electrostatic discharge, or ESD, occurs when a device is improperly handled. A human body routinely develops an electric potential in excess of 1,000 V. Upon contacting an electronic device, this buildup will discharge, which will create a large potential difference across the device. The effect is known to have catastrophic effects in circuits and could have similar effects in MEMS. While the effects of ESD on MEMS structures have not been published to date, it can be assumed that certain electrostatically actuated devices will be susceptible to ESD damage [21]. Figure 5 shows a typical ESD/EOS damage input protection circuitry and the catastrophic effect on the device [9].

Dielectric charging and breakdown is the charging that may occur in the dielectric layer. Sensors are known to drift over time due to charge accumulating at the surface [21].

3.7. Radiation Effects. The field of radiation effects on MEMS is becoming increasingly important. It has long been known that electrical systems are susceptible to radiation, and recent research has raised the possibility that mechanical devices may also be prone to radiation-induced damage. Especially sensitive to radiation are devices that have mechanical motion governed by electric fields across insulators, such as electrostatically positioned cantilever beams. Insulators can fail under single event dielectric rupture. A further complication is the fact that radiation can cause bulk lattice damage and make materials more susceptible to fracture [21]. 


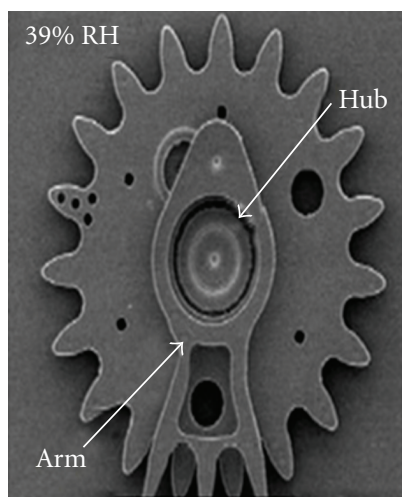

(a)

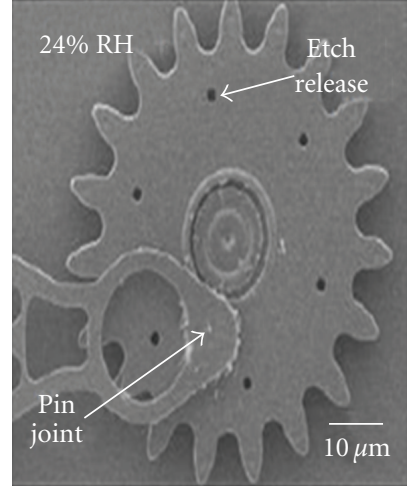

(b)

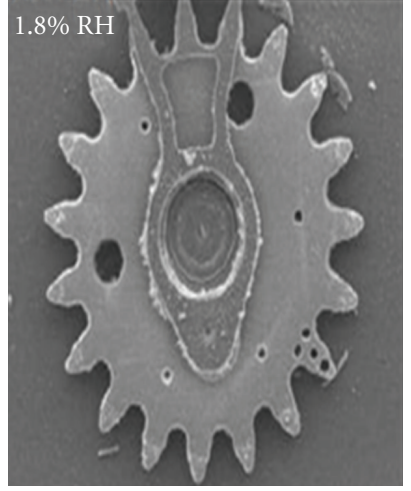

(c)

FIgURE 6: Micro engine gears under humidity stress (courtesy of Sandia National Laboratories).

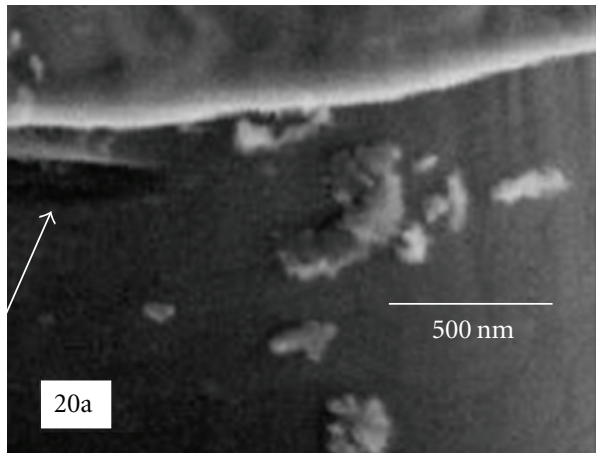

(a)

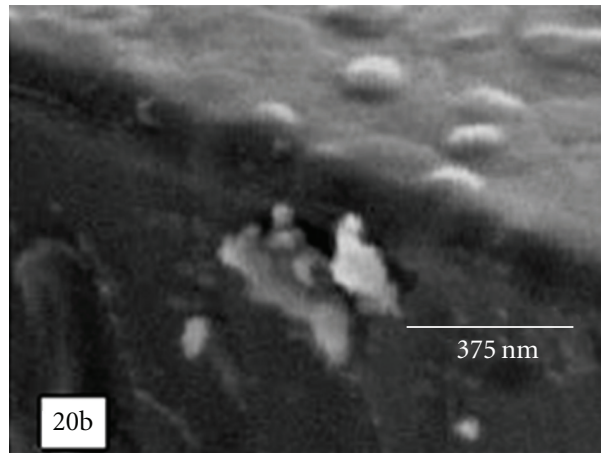

(b)

FIGURE 7: Particles on MEMS (courtesy of Sandia National Laboratories).

3.8. Temperature. This is a serious concern for MEMS. Internal stresses in devices are extremely temperature dependent. The temperature range in which a device will operate within acceptable parameters is determined by the coefficient of linear expansion. In devices where the coefficients are poorly matched, there will be a low tolerance for thermal variations [9].

Thermal effects cause problems in metal packaging, as the thermal coefficient of expansion of metals can be greater than ten-times that of silicon. For these packages, special isolation techniques have to be developed to prevent the package expansion from fracturing the substrate of the device. Another area that has yet to be fully examined is the effect of thermal changes upon the mechanical properties of semiconductors. It has long been known that Young's modulus is a temperature-dependent value $[21,28]$.

3.9. Humidity. Humidity is considered another serious concern for MEMS. Surface micromachined devices are extremely hydrophilic for reasons related to processing. In the presence of humidity, water will condense into small cracks and pores on the surface of these structures [21,29].

Figure 6 shows an experiment performed by Sandia National Laboratories to microengine gears stressed under different humidity conditions, that is, 39\%, 24\%, and $1.8 \%$ of relative humidity $(\mathrm{RH})$ at $25^{\circ} \mathrm{C}$. The microengines were stressed for the same number of cycles, but the amount of wear debris for each humidity value was dramatically different [30].

3.10. Particulates. Particulates are fine particles that are prevalent in the atmosphere. These particles have been known to electrically short out MEMS and can also induce stiction. While these particles are normally filtered out of the clean room environment, many MEMS are designed to operate outside the confines of the clean room and without the safety of a hermetically sealed package.

As a result, devices must be analyzed to ensure that they are particle-tolerant before they can be used as high-reliable devices in environments with high particulate densities. Another area in which contaminants cause problems is in adhesion. Proper device processing requires most materials interfaces to be clean in order to have good adhesion. If dust particles are present, the two materials are weakly bonded and are more likely to have delamination problems [21]. Figure 7 shows a wear experiment conducted by Sandia National Laboratories. Particles can be easily identified in 
TABLE 1: MEMS failure mechanisms.

Class I: accelerometers, pressure sensors, inkjet print heads, and strain gauges

Class II: gyroscopes, comb drives, resonator, and filters

Class III: relays and valves

Class IV: shutters, scanners, and optical switches
Failure mechanism description: unknown to fail due to operation. Particulate contamination can and typically will induce failure. Particles can be difficult to detect because they may not electrically interfere with the operation of a device. Particulate contamination may serve to mechanically obstruct the device while its electrical integrity is maintained.

Failure mechanism description: these devices have intentionally designed moveable parts that interact with the rest of the device to perform a given function, they are susceptible to fatigue, fracture, or particulate contamination.

Failure mechanism description: MEMS devices with impacting surfaces have the potential to create debris, fracture components, induce cracks, and so forth. Impact failures are very dependent upon the force exerted on the opposite MEMS structure.

Failure mechanism description: these devices have moving, impacting structures with the addition of rubbing surfaces. Rubbing creates friction and often will result in the creation of wear material or debris. The formation of this material may result in several different failure mechanisms. These are failure by particle contamination binding the device, particles causing third body wear changing the motion tolerance, particulate contamination preventing or obstructing motion, and adhesion of rubbing or contacting surfaces. The mechanism for wear may depend on the temperatures reached during rubbing. the side wall of the device, and they are responsible to start the initial wear process.

MEMS components by nature have different and unique failure mechanisms than their macroscopic counterparts. As it was mentioned before, Walraven [9] developed a general taxonomy to properly categorize each MEMS device with their related failure mechanism. Table 1 shows the result of such a categorization.

\section{Final Remarks}

Reliability for MEMS devices is identified as the next manufacturers challenge for the forthcoming years due to a growing market and stricter government safety regulations. It is necessary to understand several variables to have an approach of their behavior and functionality. Very high levels of reliability are required in most industrial applications for MEMS, hence, a good understanding of MEMS internal variables (i.e., technologies related) and external variables (i.e., environment and operation conditions). MEMS reliability analysis is extremely important to identify and understand the different failure mechanisms that can be implicit such as mechanical, thermal, and chemical aspects related to the diversity of materials that can be used.

\section{References}

[1] W. Miller, D. Tanner, S. Miller, and K. Peterson, MEMS Reliability: The Challenge and the Promise, The Reliability Challenge, Dublin, Ireland, 4th edition, 1998.

[2] W. van Spengen, "MEMS reliability from a failure mechanisms perspective," Microelectronics Reliability, vol. 43, no. 7, pp. 1049-1060, 2003.
[3] J. Walraven, "Future challenges for MEMS failure analysis," in Proceedings International Test Conference (ITC '03), pp. 850855, October 2003.

[4] B. Bhushan, Springer Handbook of Nanotechnology, Springer, New York, NY, USA, 2007.

[5] D. S. Eddy and D. R. Sparks, "Application of MEMS technology in automotive sensors and actuators," Proceedings of the IEEE, vol. 86, no. 8, pp. 1747-1755, 1998.

[6] J. Li, A. Q. Liu, and Q. X. Zhang, "Tolerance analysis for combdrive actuator using DRIE fabrication," Sensors and Actuators, vol. 125, no. 2, pp. 494-503, 2006.

[7] D. Tanner, J. Walraven, M. Irwin et al., "The effect of humidity on the reliability of a surface micromachined microengine," in Proceedings of the 37th Annual IEEE International Reliability Physics Symposium (IRPS '99), pp. 189-197, San Diego, Cailf, USA, March 1999.

[8] D. P. Vallett, "Failure analysis requirements for nanoelectronics," IEEE Transactions on Nanotechnology, vol. 1, no. 3, pp. 117-121, 2002.

[9] J. Walraven, "Failure mechanisms in MEMS," in Proceedings International Test Conference (ITC '03), pp. 828-832, 2003.

[10] D. Tanner, "MEMS reliability: where are we now?" Microelectronics Reliability, vol. 49, no. 9-11, pp. 937-940, 2009.

[11] W. Sharpe and J. Bagdahn, "Fatigue of materials used in microelectromechanical systems (MEMS)," in Proceedings of the 8th International Congress on Fatigue, Stockholm, Sweden, 2002.

[12] H. Shea, "Reliability of MEMS for space applications," in Proceedings of the SPIE, 2006.

[13] R. Ritchie, J. Kruzic, C. Muhlstein, R. Nalla, and E. Stach, "Characteristic dimensions and the micro-mechanisms of fracture and fatigue in 'nano' and 'bio' materials," International Journal of Fracture, vol. 128, no. 1, pp. 1-15, 2004.

[14] A. Fitzgerald, D. Pierce, B. Huigens, and C. White, "A General Methodology to Predict the Reliability of Single-Crystal Silicon MEMS Device," IEEE, Journal of Microelectromechanical Systems, vol. 18, no. 4, 2009. 
[15] J. Pomeroy, P. Gkotsis, M. Zhu, G. Leighton, P. Kirby, and M. Kuball, "Dynamic operational stress measurement of MEMS using time-resolved Raman spectroscopy," Journal of Microelectromechanical Systems, vol. 17, no. 6, pp. 1315-1321, 2008.

[16] O. Jadaan, N. Nemeth, J. Bagdahn, and W. Sharpe, "Probabilistic Weibull behavior and mechanical properties of MEMS brittle materials," Journal of Materials Science, vol. 38, no. 20, pp. 4087-4113, 2003.

[17] L. Starman, J. Busbee, J. Reber, J. Lott, W. Cowan, and N. Vandelli, "Stress measurement in MEMS devices," in Proceedings of the International Conference on Modeling and Simulation of Microsystems (MSM '01), pp. 398-401, 2001.

[18] U. Schwalke, M. Pölzl, T. Sekinger, and M. Kerber, "Ultra-thick gate oxides: charge generation and its impact on reliability," Microelectronics and Reliability, vol. 41, no. 7, pp. 1007-1010, 2001.

[19] W. Luo, D. Sunardi, Y. Kuo, and W. Kuo, "Stress stress testing and characterization of high-k dielectric thin films," in Proceedings of the IEEE International, Integrated Reliability Workshop Final Report, 2003.

[20] S. Brown, W. van Arsdell, and C. Muhlstein, "Materials reliability in MEMS devices," in Proceedings of the 1997 International Conference on Solid-State Sensors and Actuators, vol. 1, pp. 591-593, June 1997.

[21] B. Stark, MEMS Reliability Assurance Guidelines for Space Applications, National Aeronautics and Space Administration (NASA) and Jet Propulsion Laboratory (JPL), California Institute of Technology, Pasadena, California, USA, 1999.

[22] W. Merlijn van Spengen, "MEMS reliability from a failure mechanisms perspective," Microelectronics Reliability, vol. 43, no. 7, pp. 1049-1060, 2003.

[23] M. Sheehy, J. Punch, S. Goyal, M. Reid, M. Lishchynska, and G. Kelly, "The failure mechanisms of micro-scale cantilevers under shock and vibration stimuli," Strain, vol. 45, no. 3, pp. 283-294, 2009.

[24] K. M. Delak et al., "Analysis of manufacturing scale MEMS reliability testing," in Proceedings of the MEMS Reliability for Critical and Space Applications, vol. 3880, SPIE, Santa Clara, Cailf, USA, Sept. 1999.

[25] A. DiBenedetto, The Structure and Properties of Materials, McGraw-Hill, New York, NY, USA, 1967.

[26] G. Subhash, A. Corwin, and M. P. de Boer, "Evolution of wear characteristics and frictional behavior in MEMS devices," Tribology Letters, vol. 41, no. 1, pp. 177-189, 2011.

[27] C. L. Muhlstein, E. A. Stach, and R. O. Ritchie, "A reactionlayer mechanism for the delayed failure of micron-scale polycrystalline silicon structural films subjected to high-cycle fatigue loading," Acta Materialia, vol. 50, no. 14, pp. 3579-3596, 2002.

[28] J. Weiss, "Magnetic force and thermal expansion as failure mechanisms of electrothermal MEMS actuators under electrostatic discharge testing," Journal of Applied Mechanics, vol. 74, no. 5, pp. 996-1005, 2007.

[29] X. D. Wang, J. X. Yu, L. Chen, L. M. Qian, and Z. R. Zhou, "Effects of water and oxygen on the tribochemical wear of monocrystalline $\mathrm{Si}(100)$ against $\mathrm{SiO}_{2}$ sphere by simulating the contact conditions in MEMS," Wear, vol. 271, no. 9-10, pp. 1681-1688, 2011.

[30] Sandia National Laboratories, Report: MEMS Reliability: Infrastructure, Test Structures, Experiments, and Failure Modes, Livermore, Cailf, USA, 2000. 

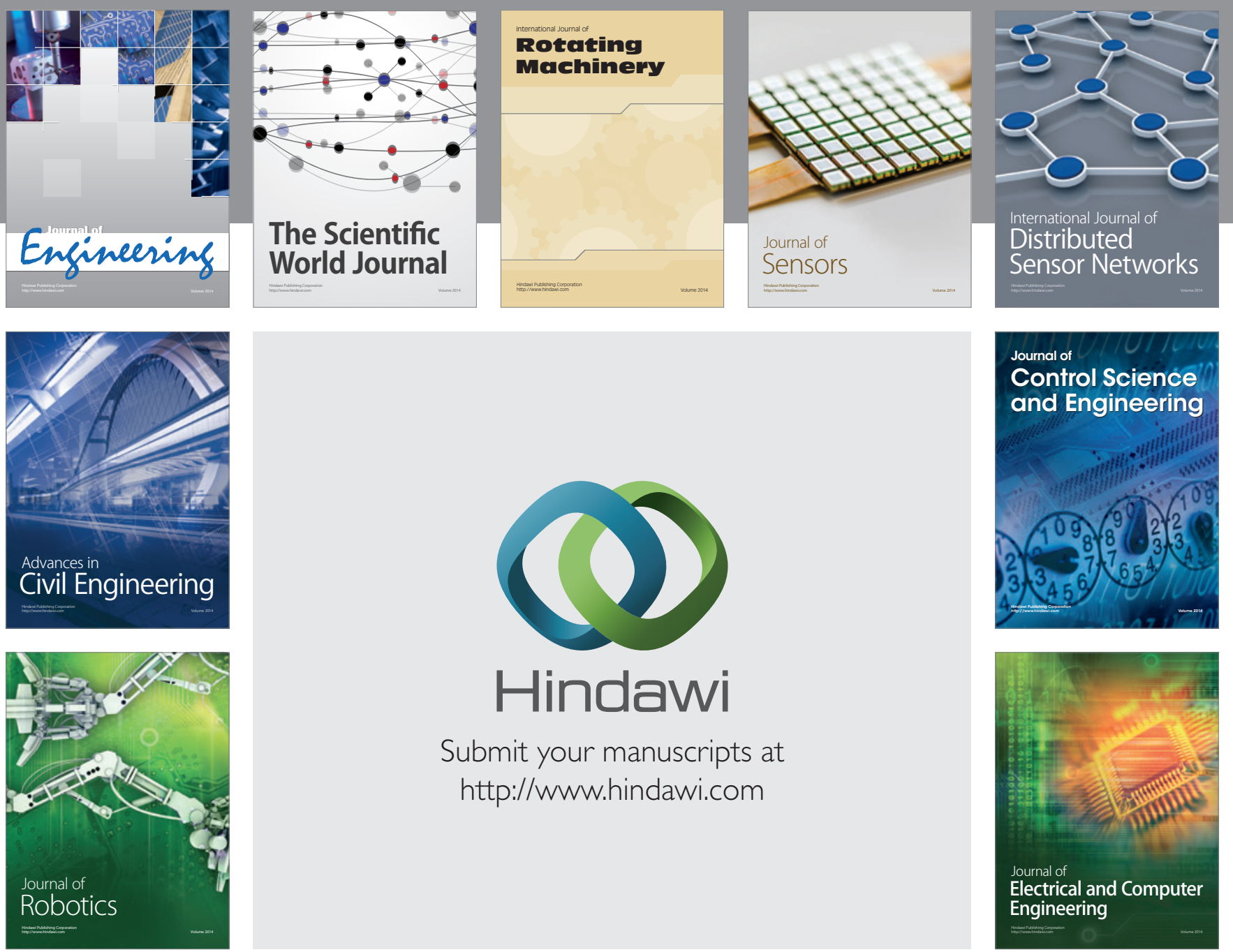

Submit your manuscripts at

http://www.hindawi.com
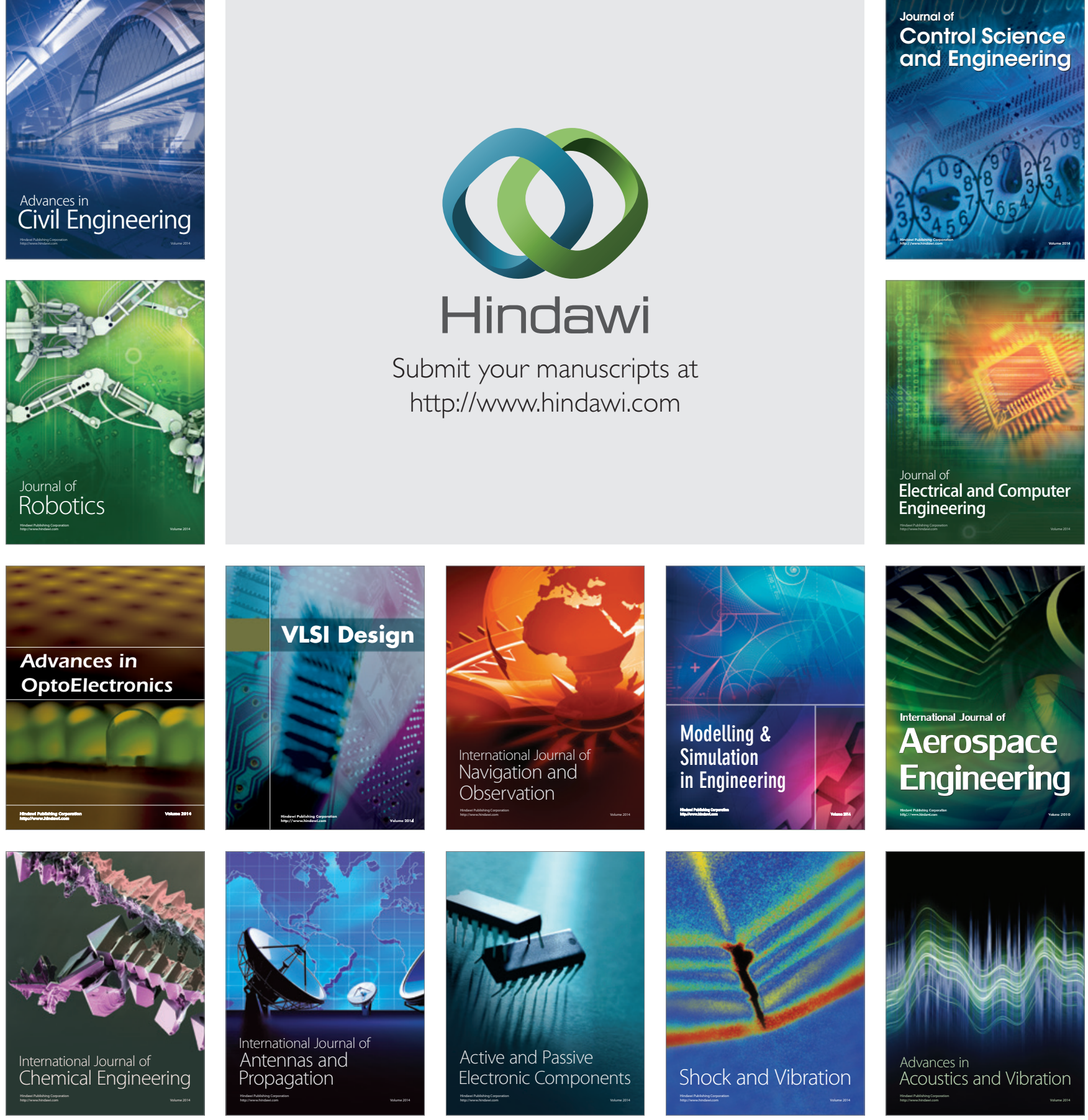